\title{
Weintourismus und damit einhergehenden Erwartungshaltungen in Deutschland
}

\author{
Jens Rüdiger, Jon H. Hanf, und Erik Schweickert \\ Hochschule Geisenheim University, 65366 Geisenheim, Germany
}

\begin{abstract}
Durch die allgemeine Zunahme von Kurzurlaubsreisen sieht auch die Weinbranche in Europa immer mehr die wirtschaftlichen Vorteile und die damit einhergehenden positiven Synergien zwischen Wein und Tourismus. Das in unseren Breiten noch sehr junge Thema Weintourismus beschränkt sich in wissenschaftlichen Arbeiten meist auf die Angebotsstruktur und nicht auf die Erwartung der Konsumenten. In dieser Untersuchung wurden die Erwartungen von Weintouristen analysiert, um die aus der Literatur und die durch Experteninterviews gewonnenen Erkenntnisse für Weinbaugemeinden als Empfehlung zu Grunde legen zu können. Es zeigte sich, dass es nur eine geringe Zahl von Weinreisenden gibt, die explizit des Weines Willen in eine Region reist. Der Großteil der Touristen möchte im Kurzurlaub vom Thema Wein am Rande in Form eines Zusatzerlebnisses berührt werden. Es zeigt sich, dass die Erwartungen an diese Erlebnisse eher klassisch und traditionell geprägt sind. Primär stehen für den Touristen Entspannung und Genuss im Vordergrund, wobei sie offen sind, um "Neues Kennenzulernen" und ihr Wissen über Wein durch kommunikative Vermittlung zu erweitern. Die Präferenz liegt auf Weinfeste und Weinveranstaltungen in Verbindung mit einem Erlebniswert. Erwartet werden Angebote an erklärten Weinverkostungen, Gemeinschaftsvinotheken und Gastronomie, die einen direkten Bezug zu Wein
\end{abstract} aufweist.

Wine tourism is a market with increasing attraction. Reason for this is that in the context of recreational activities and tourism wine and its production are gaining popularity among Germans. However, the majority of tourists, for who wine is of interest during a getaway, only want to be briefly touched upon this topic. These wine tourists are mainly looking for relaxation and pleasure. Nevertheless, they are open to get to know something new and to learn something about wine. The response on the question "which expectations has a wine tourists on the offer of a wine growing community?" shows that rather expectations can be described as classical and traditional. The main expectation is that wine tourists want to have a great experience. The marketing of a wine destination can only be successful if the community and the local wine business are cooperating. The distribution of strategic and operative tasks is depending on the financial and economical situation as well as human resources and the motivation that the participating parties have.

L'oenotourisme est un marché gagnant de plus en plus d'intérêt, car le vin et sa production augmente dans l'estime des Allemands quant à leurs loisirs et en ce qui concerne les vacances et le tourisme. Mais c'est un fait qu'une grande partie des touristes pour lesquels le vin est intéressant dans le cadre d'un court temps libre ne veut être touchée par ce sujet qu'en passant. Ce sont la détente et la jouisssance qui comptent avant tout pour les touristes, ceux-ci étant ouverts à « la connaissance du nouveau » et à l'élargissement de leur savoir sur le vin dans une entremise communicative. En réponse à la question «Quelles attentes l'oenotouriste a-t-il envers l'offre d'une commune vinicole ? ", les enquêtes quantitatives montrent que les attentes des oenotouristes sont plutôt classiques et traditionnelles. Il faut retenir que les touristes du vin - quand ils entrent dans un site vinicole - veulent faire l'expérience d'une découverte. Cependant, la commercialisation d'une telle destination ne peut être couronnée de succès que dans une coopération entre la commune et l'économie vitivinicole. La répartition de la mis en œuvre du programme stratégique et opérationnel dépend de la situationu financière, économique et personnelle et de la motivation des partenaires concernés.

\section{Einleitung}

Weintourismus ist für die Tourismuswirtschaft weinbautreibender Länder ein Markt der Zukunft und nicht nur der Tourismusminister von Südafrika konstatiert: "Weintourismus ist eine wichtige Sparte in Südafrikas Tourismusangebot". Die von der internationalen Fachmesse für Weine und Spirituosen "ProWein" initiierte Verbraucherumfrage belegt: Gerade bei der älteren Zielgruppe besteht ein gesteigertes Interesse für mit
Wein verbundene Freizeitangebote mit Weingut-Besuchen - vorzugsweise in Deutschland (49 Prozent), Frankreich (32 Prozent) und Italien (29 Prozent), aber auch Spanien, Österreich, Ungarn, Südafrika und die USA liegen bei deutschen Touristen mit Interesse für Wein im Trend. 57 Prozent der Befragten haben schon mal ein Weingut besucht, 78 Prozent haben schon an einer Weinprobe teilgenommen, bei den über 50jährigen sogar 86 Prozent. Das zeigt: Weintourismus liegt im Trend und Wein und seine Herstellung steigen im Interesse der Deutschen bei 
der Freizeitgestaltung und in Verbindung mit Urlaub und Tourismus [1].

Aber was genau macht dieses Interesse bei Weintouristen aus und was erwarten sie, wenn sie als Touristen aus Interesse für Wein einen Ort bereisen? Der Schwerpunkt der bisherigen Forschung liegt hier eher auf Fragen zur touristischen Angebotsstruktur und zur Bereisung bestimmter Regionen.

Was aber genau erwartet der an Wein interessierte Tourist beim Besuch einer Destination? Gibt es Unterschiede in dieser Erwartungshaltung bei "normalen" Touristen und an Wein interessierten Touristen und wenn ja: Was unterscheidet den Weintouristen von allen anderen Touristen und woraus besteht seine Erwartungshaltung? Antworten auf diese Fragen finden sich kaum in der Literatur. Ziel dieser Arbeit war es, diese Erwartungshaltung von Weintouristen anhand einer Evaluation zu definieren und zu erörtern.

\section{Methodisches Vorgehen}

$\mathrm{Zu}$ Beginn der Arbeit wurden zunächst begriffliche Grundlagen wie Weintourismus, Weintourist, Destination, Kurzurlaub und Erwartungshaltung abgesteckt, um die in der Arbeit verwendeten Definitionen zu Grunde zu legen. Bereits hier wurde festgestellt, dass es in der Literatur sehr weitläufige und unterschiedliche Definitionen von "Weintouristen" gib. Die in der Literatur gängigen Definitionen wurden in Kapitel 3.4 gegenübergestellt mit dem Ergebnis eines sehr breiten Spektrums an unterschiedlichen Definitionen. Deshalb wurde in diesem Kapitel zunächst ein eigener Begriff von Weintouristen definiert, um damit den genauen begrifflichen Rahmen für das weitere Forschungsvorhaben zu schaffen.

Als zweiter Schritt wurde eine qualitative Erhebung durch Experteninterviews angesetzt. Um möglichst breitgefächerte Einblicke in das Thema zu erhalten und unterschiedliche Blickwinkel einzubinden, wurden zehn Interviews mit Experten geführt, die in den für die Befragung vier maßgeblichen Organisationsfeldern des Themengebietes "Wein" tätig sind: Verbände, staatliche Institutionen, Wissenschaft und Journalismus. Die Befragten wurden aufgrund ihrer Funktion in den jeweiligen Organisationen ausgewählt. Dafür wurde für die Experteninterviews ein einheitlicher Interviewleitfaden verwendet, um eine vergleichbare Auswertung der Interviews sicherzustellen. Die Interviews wurden im Anschluss transkribiert und nach der formulierten Fragestellung "Wie wichtig ist die Thematik Wein für einen Touristen, ein spezifisches Ziel zu besuchen und welche Erwartung ist damit verbunden?" ausgewertet. Dabei wurden die Expertenaussagen zur Erwartungshaltung von Weintouristen in die vier Erlebnissphären nach Pine und Gilmore [2] kategorisiert.

\section{3. Überblick aus der Literatur}

Obwohl sich in der englischsprachigen Literatur unterschiedliche Definitionen zum Begriff Weintourismus finden, existiert in der Tourismuswissenschaft noch immer keine allgemeingültige Definition [3,4]. Insgesamt ist festzustellen, dass in Australien, Neuseeland und
Südafrika eine intensivere wissenschaftliche Auseinandersetzung mit der Thematik des Weintourismus erfolgt.

Aufgegriffen wurde der Begriff Weintourismus erstmals zu Beginn der 1990er Jahre von der Australian Tourist Commission [3]. Die von den meisten Autoren verwendete Definition für Weintourismus stammt von C. M. HALL aus den Jahre 1996: "Visitation to vinyards, wineries, wine festivals, and wine shows for which grape wine tasting and/or experiencing the attributes of a grape wine region are the prime motivating factors for visitors" [5]. Im Jahr 2000 wurde diese Definition in Bezug auf die Teilnehmer und die Destination des Weintourismus erweitert. Demnach ist Weintourismus eine "tourism activity influenced by the physical, social and cultural dimensions of the winescape and its components" [6].

Müller und Dreyer [3] beschreiben, wie diese Definition von vielen Autoren aufgegriffen und ergänzt wird und stellen daraus folgende gemeinsame Definitionsmerkmale heraus:

- die Motivationsgrundlage des Reisenden ist die Weinregion und sind die weinbezogenen Erlebnisse vor Ort

- der Bezug zu Wein ist in der Destination gegeben

- Wein steht in der touristischen Aktivität vor Ort im Vordergrund

- zwischen Wein- und Kulturtourismus besteht eine enge Verbindung

- es besteht eine Kooperation verschiedener Akteure im Weintourismus.

\subsection{Synergien zwischen Wein und Tourismus}

Beim Weintourismus handelt es sich um eine Kombination der beiden Branchen Tourismus und Wein [7]. Beide können, da Weintourismus ein Synergiefeld zwischen Weinbau und Tourismus darstellt, voneinander profitieren [3]. Allerdings herrscht unter den Vertretern beider Branchen Misstrauen bzw. fehlt das Verständnis für den jeweils Anderen. Auch deshalb befindet sich dieser Zweig des Tourismus noch in seiner Aufbauphase [7]. Um die positiven Synergien besser nutzen zu können, muss das gegenseitige Verständnis gestärkt werden. Dazu ist es grundlegend wichtig, dass beiden Seiten die Sichtweise und Strategie des jeweils Anderen näher gebracht wird [8] denn der Nutzen der Zusammenarbeit und die Vernetzungsmöglichkeiten von unterschiedlichen Akteuren im Weintourismus ist vielfältig [9]. Wein und Natur, Architektur, Aktivtourismus, Genuss und Kultur [8] sind ausbaufähige Synergieeffekte. Doch auch Professionalität und Entwicklungsstand der ansässigen Akteure sind ausschlaggebend für erfolgreichen Weintourismus. Hinzu kommt als grundlegende Voraussetzung, Weintouristen für die jeweilige Destination zu gewinnen, eine ausreichende Qualität der Weine und das Engagement der Tourismuswirtschaft [7].

\subsection{Die Destination als gemeinsames Handlungsfeld von Wein und Tourismus}

In der geographischen Wissenschaft ist Wein der Agrargeographie und Tourismus der Tourismusgeographie 
zugeordnet. Aus den gemeinsamen Handlungsfeldern ergibt sich der Weintourismus [10]. Die gemeinsame Komponente von Wein und Tourismus ist der Bezug zur räumlichen Einheit. Diese spiegelt sich bei der Weinproduktion in einem geographisch abgegrenzten Raum wieder, der nach Strecker [10] auch eine touristische Destination definiert. Eine Destination ist ein "geographischer Raum, den der jeweilige Gast als Reiseziel auswählt. [...] Es ist das jeweilige, für eine bestimmte Zielgruppe relevante Zielgebiet" [11].

Die betriebswirtschaftliche Literatur beschreibt eine Destination auch als "virtuelle Unternehmen", da eine gemeinschaftliche Leistung für Dritte erstellt wird. So werden innerhalb der Destination von den Leistungsträgern einzelne touristische Elemente erbracht und zu einem Leistungsbündel geschnürt. Dieses wird vom Tourist als Ganzes wahrgenommen [12]. Die Vermarktung einer Destination ist daher ein wesentlicher Aspekt, um Synergieeffekte auszuschöpfen und eine enge Kooperation zwischen unterschiedlichen Leistungsträgern zu ermöglichen [8].

Innerhalb einer Destination sind zahlreiche verschiedene Partner nötig, die sich jedoch häufig als ein eigenständiger Zweig einer separaten Industrie betrachten. Gerade Weingütern sind die Vorteile des Weintourismus häufig noch nicht ausreichend bewusst, denn es herrscht eine starke Fixierung auf das eigene Produkt [3]. Doch jedes Weingut ist durch seine Existenz bereits ein Teil des Weintourismus einer Destination. Das Engagement, am Tourismus vor Ort teilzunehmen, wird jedoch von unterschiedlichen Faktoren wie Größe des Weinguts, Absatzmarkt, Geschäftsstrategie und persönliche Entscheidung bestimmt [5]. Außerdem können andere Hemmnisse wie eine unzureichende Infra- und Angebotsstruktur, fehlende kommunal- und privatwirtschaftliche Initiativen, Veränderungen des Landschaftsbilds in Form von Brachflächen sowie mangelnde Akzeptanz und Identifikation der einheimischen Bevölkerung eine erfolgreiche Entwicklung des Weintourismus innerhalb einer Destination verhindern [13].

\subsection{Zielgruppen im Weintourismus}

Für eine Marktbetrachtung ist die Analyse der Nachfrageseite im Weintourismusbereich unerlässlich [14]. Dennoch ist die Erforschung der weintouristischen Zielgruppe noch sehr jung. Erst Mitte der 1990er Jahre ist der Weintourist in den Mittelpunkt der Forschung gerückt. Zuvor wurde in Untersuchungen meist die Angebotsseite betrachtet [15].

Hall et al. [5] beschreibt einen Weintourist als einen regelmäßigen Besucher von Weinveranstaltungen, Weingütern und Weinbergen mit dem Ziel, sich zu erholen. Er ist mehrheitlich zwischen 30 und 50 Jahre alt und verfügt über ein mittleres bis hohes Einkommen. Die psychographischen Eigenschaften eines Weinreisenden zeigen, dass er über gute Kenntnisse zum Thema Wein verfügt und diesen regelmäßig konsumiert. Charters/ Ali-Knight [16] beschreiben den Weintourist als jemanden, der schon einmal irgendeine Art von Weinerlebnis genossen hat, sei es in Form von Literatur, Film oder aus persönlichen Erfahrungen. Allgemein treten Besucher einer Weinregion laut Scheffel [17] häufig als Reisegruppen auf, weniger als Einzelreisende. Die durchschnittliche Gruppengröße beträgt 3,5 Personen.

Da sich die ersten schwerpunktmäßigen Untersuchungen zum Weintourist hauptsächlich auf die "Neue Welt" beziehen, sind obengenannte Aussagen jedoch kaum auf alle Weintourismusgebiete übertragbar [10]. Dies lässt sich auch in den länderspezifischen Besonderheiten des Weinkonsums beobachten [3]. Wichtig ist allgemein bei der Zielgruppendefinition einer Weinregion, dass keine potenziellen Nachfrager ausgeschlossen werden [18] und Reisemotive, die in weintouristische Destinationen führen, nicht nur vom Wein geprägt sind, sondern der Reiseentscheidung ein Motivbündel zu Grunde liegt. Dazu werden in der Literatur unterschiedliche Motivationsfaktoren aufgezeigt, die als entscheidungsrelevant gelten [3]. So können Beweggründe durch externe und interne Faktoren geprägt sein. Bei externer Motivation wird das Reisen mit einer konkreten Zielsuche verbunden. Diese wird mit der Charakteristika und/oder Aktivitäten einer Weinbauregion verbunden. Verkostung oder Kauf von Wein, aber auch Touren durch die Weinberge sind Beispiele dafür. $\mathrm{Zu}$ den internen Motivationsfaktoren zählt hauptsächlich der Urlaub als Ausgleich zum Alltag mit dem Wunsch nach Erholung [15]. Andere häufig genannte Motivationsfaktoren für den Weintouristen sind auch persönliche Kenntnisse zu erweitern, Teilnahme an Kellerführungen, Treffen mit dem Winzer und Essen auf dem Weingut [14,15]. Dabei ist die Bereitschaft, weintouristische Angebote $\mathrm{zu}$ nutzen, bei Weinkonsumenten prinzipiell höher, als bei weinuninteressierten Konsumenten [19]. Saaler [20] verweist auf eine Studie von Thach, nach der es zehn Motive gibt, die für eine Weinreise ausschlaggebend sind: Erweitern von Weinkenntnissen, Verköstigung von Wein, Erlebnisse vor Ort, ländliche Umgebung, Zusammenspiel von Wein und Kulinarik, kulturelle Aspekte, Kunst und Weinarchitektur, Veranstaltungen im Weintourismus, gesundheitliche Aspekte und das Kennenlernen nachhaltiger Komponenten im Weintourismus.

Innerhalb des Weinmarktes lässt sich der Weintourist aufgrund seiner Reisemotivation in drei unterschiedliche Segmente aufteilen: den Weininteressierten, den Weinurlaubsaffinen und den "echten" Weinreisenden.

\subsection{Kurzzeittourismus als Urlaubsform für Weintourismus}

Tourismus ist national und international eine ausgeprägte Wachstumsbranche. Auch für die Zukunft wird nicht mit einem Einbruch dieser Entwicklung gerechnet [11]. Hinzu kommt, dass sich das Reiseverhalten der Deutschen verändert. Die Hauptreisedauer und die Dauer von zusätzlichen Urlaubsreisen nahmen in den letzten Jahren stark ab, jedoch vermehrt sich die Zahl der Reisen [21]. Aus touristischer Sicht ist die Reisedauer als weiterer Faktor für eine Zielgruppe zu definieren [22].

Allgemein geht man von einer ortsverändernden Reise mit einem vorübergehenden Aufenthalt von mindestens einer Übernachtung aus [23]. Bleile [24] definiert Kurzurlaub als Aufenthalt mit einer Reisedauer von zwei bis vier Tagen - häufig eine spontane Reise über ein Wochenende. Im Jahr 2011 wurden neben etwa 


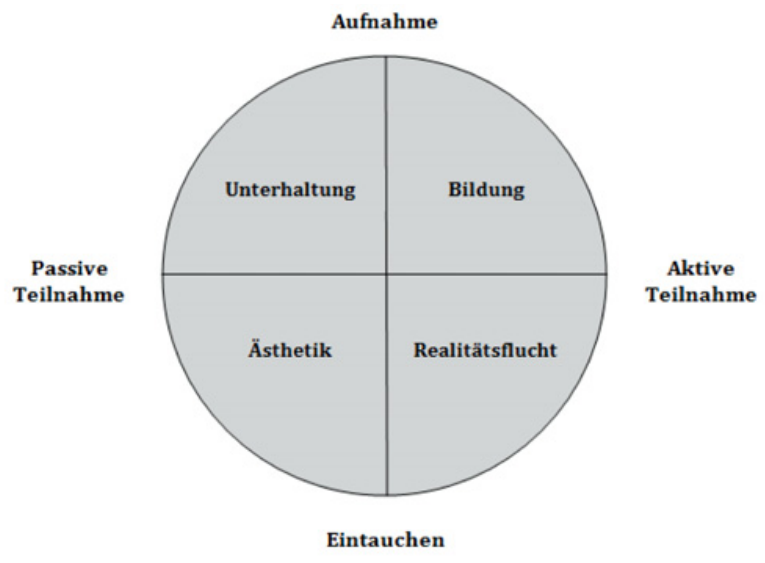

Abb 1. Erlebnissphären nach Pine und Gilmore Quelle: Eigene Darstellung in Anlehnung an Pine/Gilmore, 1999, S. 30.

70 Millionen Urlaubsreisen auch etwa 78 Millionen Kurzurlaubsreisen von deutschen Touristen unternommen [21]. 2012 unternahmen 75,6 Prozent der deutschen Kurzurlauber ihre Reise in Deutschland [25]. Daher werden verstärkt regionale Weintourismuskonzepte, wie z.B. das von der Region Franken, auf Touristen mit einer Reisedauer von einem bis drei Tagen ausgelegt [22].

\subsection{Die Erwartungshaltung von Touristen}

Nach Hillmann [26] ist die Erwartungshaltung ein "Grundbegriff der soziologischen Rollentheorie [und; $J . R$.] bezeichnet den jeder sozialen Beziehung zugrunde liegenden Tatbestand, dass aus den sozialen Rollen der betreffenden Handelnden bzw. aus den mit der Rollenübernahme verbundenen Ansprüchen oder Einstellungen bestimmte Aktions- oder Reaktionsformen der Beteiligen untereinander abgeleitet werden können". Bereits Max Weber, definiert in anderen Worten Erwartungen als "ein seinem Sinngehalt nach aufeinander eingestelltes und dadurch orientiertes Sichverhalten mehrerer" [26]. In Bezug auf die Erwartungshaltung in der Tourismuswirtschaft stellt Luft [27] fest, dass alle Leistungsträger der Erkenntnis unterliegen, dass touristische Produkte sich aufgrund der multioptionalen Erwartungshaltung der Touristen durch die Austauschbarkeit verschiedener Teilleistungen und Gegebenheiten auszeichnen. Bleile [24] konkretisiert, dass durch gestiegenen Wohlstand, höheren Wohnkomfort und zunehmende Reiseerfahrung der Anspruch und die Erwartungshaltung an die Urlaubsdestination in den letzten 20 bis 25 Jahren gestiegen ist. Diese gilt es zu erfüllen. Swarbrooke [28] stellte als wichtig heraus, dass Anbieter auf spezielle Kundenwünsche eines Kundensegmentes oder einer Zielgruppe eingehen.

Zusammenfassend gilt für eine Weinregion: Qualität ist die Fähigkeit einer Region, Erwartungen zu erfüllen, die in sie gesetzt werden. Umgekehrt heißt dies, dass Weinregionen oder Weinbaubetriebe nur dann für Qualität stehen, wenn sie den Ansprüchen und Erwartungen der Gäste gerecht werden [29]. Wie kann jedoch überprüft werden, ob eine Weinregion diesen Ansprüchen
Rechnung trägt? Die Theorie von Pine und Gilmore der Erlebnisökonmie mit ihren vier Erlebnisphären wird im folgenden Abschnitt dargelegt. Sie ist theoretische Grundlage für die Überprüfung von Erwartungen durch die quantitative Erhebung.

\subsection{Die vier Erlebnissphären von Pine und Gilmore}

In Deutschland entwickelt sich Weintourismus zu einem stark wachsenden Markt. Mit immer mehr Erlebnisangeboten investieren Weinregionen und Weinbaubetriebe in den Tourismus [30]. Im Freizeit- und Tourismusbereich ist die Erlebnisorientierung jedoch kein neues Konzept. Das Erlebnis steht im "neuen" Tourismus als eigentlicher Nutzen immer schon im Mittelpunkt der Kundennachfrage [31]. Durch den Erwerb eines Erlebnisses wird der Aufenthalt einer touristischen Dienstleistung mit unvergesslichen Ereignissen ausgefüllt, da der Tourist persönlich in das Erlebnis eingebunden wird. Dies ist der Fall, wenn eine Dienstleistung auf einer Inszenierung basiert. Pine und Gilmore definieren das als Erlebnisökonomie. Dabei geht es darum, den Gast nicht nur zu unterhalten sondern ihn mit einzubeziehen. Erfolgreiche Unternehmen erzielen für ihre Produkte einen höheren Preis wenn sie die Fähigkeiten eines Produktes in den Vordergrund rücken und wenn es ihnen gelingt, damit Erlebnisse in den Konsumenten hervorzurufen. Als Fazit muss für eine erfolgreiche Erlebnisökonomie eines Unternehmens die Kommunikation von Erlebniswerten im Mittelpunkt stehen [2].

Mit den vier Erlebnissphären von Pine und Gilmore (vgl. Abbildung 1) wird ein Weg zur Kundenansprache und zur Entwicklung erlebnisorientierter Angebote aufgezeigt. In ihrem Konzept werden mit Achsen zwei Dimensionen unterschieden, die gekennzeichnet sind durch die aktive oder passive Teilnahme sowie die Aufnahme oder das Eintauchen.

Die horizontale Achse beschreibt die Teilnahmeaktivität des Gastes. Die rechte Seite ist dabei durch die aktive Teilnahme geprägt, die linke Seite erfordert keine aktive Teilnahme. Die Achse in der vertikalen Stellung bezieht sich auf das Involvement des Gastes. Der obere Teil zeigt die Aufnahme, die für Aufmerksamkeit steht, das Erlebnis bleibt in Erinnerung. Im unteren Teil taucht der Gast ein und fühlt sich als Teil des Erlebnisses. Die daraus erarbeiteten vier Sphären stellen die verschiedenen Möglichkeiten des Erlebens dar:

- Unterhaltung: Ist durch eine passiv aufnehmende Haltung geprägt

- Bildung: Wird im Gegenzug zur Unterhaltung durch aktive aufnehmende Teilnahme gekennzeichnet

- Realitätsflucht: Der Gast taucht sehr stark in das Geschehen ein und beteiligt sich aktiv

- Ästhetik: Der Gast nimmt das Erlebnis passiv auf und nimmt keinen Einfluss darauf.

Erlebnisse, die alle vier Sphären umfassen, sind für die Kundenzufriedenheit dabei am wirkungsvollsten [2]. 


\section{Die empirische Untersuchung durch qualitative Interviews}

\subsection{Erstellung des Fragebogens für die qualitative Befragung}

Für die qualitativen Interviews wurde ein standardisierter Interviewleitfaden formuliert. Dieser ermöglichte einen konstanten Ablauf der Befragung bzw. die Vergleichbarkeit der Auswertung der Interviews. Bei einzelnen Fragen wurden - je nach Antwort des Interviewten - ergänzende Unterfragen gestellt und im Laufe des Gesprächs dem Aussageinhalt angepasst. Dabei wurde auf einen flüssigen Gesprächsablauf geachtet.

In Bezug auf das Fragedesign wurden offenen Fragen gewählt, um bei den Befragten eine möglichst große Bandbreite der Antworten zu generieren. Inhaltlich haben sich die Fragen an der Grundfragestellung und an den in der Literatur diskutierten Forschungsfragen orientiert: Wie ist die generelle Erwartungshaltung von Weintouristen? Wie ist die Erwartungshaltung an eine Destination? Wer ist der Weintourist? Wie haben sich die Erwartungshaltung und die Erfüllung der Erwartungshaltung von einer Destination verändert?

Weiter wurden Aussagen in Bezug auf die Aufgabenverteilung innerhalb der Destination, die die Erwartungshaltung befriedigen, gesammelt, um diese Erkenntnisse bei einer praktischen Umsetzung in der Zieldestination einfließen lassen zu können.

Um möglichst spontane Antworten zu erhalten, wurde den Teilnehmern der Fragenkatalog nicht vorab zugeschickt. Die Befragungen der Experten wurden mit einer Vorstellungsrunde und Informationen zum Hintergrund des Interviews begonnen und mit dem Einverständnis der Befragten als Audiodatei aufgezeichnet. Von den Interviewpartnern wurde nach Beendigung der Gespräche eine schriftliche Einverständniserklärung zur Datenverwertung eingeholt. Im Anschluss an die Interviews wurden die Aufzeichnungen jeweils zeitnah wörtlich dokumentiert und transkribiert.

\subsection{Auswertung der Experteninterviews}

Die qualitativen Interviews lieferten umfassende Informationen zur genannten Forschungsfrage. Nach Abschluss der Interviews und deren Auswertung ergab sich eine Konkretisierung der Fragen:

a.) Wie wichtig ist die Thematik Wein, ein spezifisches Ziel zu besuchen, für einen Touristen und wer ist der Tourist?

b.) Wie ist die Aufgabenverteilung innerhalb einer Destination, um diesen Erwartungen gerecht $\mathrm{zu}$ werden?

c.) Welche Erwartungshaltung hat ein Weintourist gegenüber dem Angebot einer Weinbaugemeinde?

Im Laufe der qualitativen Experteninterviews wurde deutlich, dass die Forschungsfrage a.) zusammen mit den aus der Literatur erworbenen Kenntnissen beantwortet werden kann. Die Frage b.) konnte grundlegend nicht geklärt werden, allerdings kristallisierten sich parallel zwei Meinungsbilder mit im Grundsatz gleicher Richtung heraus. Bei der Frage der Erwartungshaltung von Weintouristen (Frage c.) zeigten die Experten unterschiedliche Ansätze auf.

\subsubsection{Kriterien für Kurzurlauber in einer Weindestination}

Die Erwartungshaltung beschreibt die Erwartung oder Annahme einer Person oder eines Handelnden darüber, was ein anderer oder mehrere andere tun würden [32]. Deshalb wurden im ersten Schritt der Auswertung "die Weintouristen" als Personengruppe der Handelnden eingegrenzt.

Alle Interviewpartner zeichnen in ihren Aussagen ein ähnliches Bild: Es gibt den "klassischen Weinreisenden", der wegen des Weins gezielt in eine Region fährt. Allerdings macht dies im Ganzen gesehen beim Tourismus nur den kleineren Teil aus. Der Großteil der Reisenden sieht Wein und Weinerlebnisse als Randerscheinung seines Urlaubs. Folgerichtig decken sich die Aussagen der Experten auch dahingehend, dass die Zielgruppe einer Weinbaugemeinde eher im Bereich des weinaffinen Touristen zu suchen ist, da dieser eine Region aufgrund der Landschaft, sportlichen Gegebenheiten, Ausflugsziele, Ruhe, Entspannung usw. aufsucht. Eine Weinbaugemeinde hat hier durch ein weinspezifisches Begleitprogramm Potential, Touristen mit diesem Programm zu locken und so "Wein" mit anderen Freizeitgestaltungen zu kombinieren.

Expertenaussagen zu Folge gibt es vor allem zwei Kategorien von Touristen, die ihren Kurzurlaub in einer Weindestination verbringen:

- “...da gibt's natürlich denjenigen, der vor allem wegen des Weines reist, der will wirklich Wein erleben. Aber das ist 'ne relativ kleine Anzahl... Es fährt also jetzt niemand dahin, weil er sagt: So, ich will jetzt Wein einkaufen. Das ist zwar ein kleiner Seitenaspekt, aber was er sucht, ist dieses Weinerlebnis außen rum, das Wandern durch die Weinberge, die gute Küche, die es gibt, die mit Wein verbunden ist..." (Experte aus dem Bereich Verbände, 2013)

- “...Also aus ein, aus ein paar Untersuchungen wissen wir, dass es gibt Wein, wirklich Weintouristen, aber diese, die Touristen, die nur wegen des Themas Wein in eine Region reisen, sind in der Größenordnung von einem Prozent, der... Der Großteil der Weintouristen die am Thema Wein grundsätzlich interessiert sind, geht in eine Weinregion, weil sie die Kombination suchen." (Experte aus dem Bereich staatliche Institutionen, 2013)

- “...Es gibt Touristen die unterschiedliche Dinge suchen, keinesfalls sind Touristen nur in Sachen Wein unterwegs..." (Experte aus dem Bereich der Wissenschaft, 2013)

- “...zwei wesentliche Gruppen zumindest herausarbeiten, das eine, also wenn das, wenn Wein ein wesentliches Motiv ist, ja. Als Nebenmotiv is noch mal 'ne andere Frage..." (Experte aus dem Bereich Wissenschaft, 2013)

Aufgrund dieser Aussagen und der Ergebnisse der Literaturrecherche zur Charakterisierung des Weintouristen 
wird der Tourist, der eine Weindestination aufsucht, in dieser Arbeit in zwei Kategorien eingeteilt:

1.) Der Weinreisende: Dabei handelt es sich um einen Weinkenner und Weinliebhaber, der gezielt Zeit in einer Weinregion verbringt, um mehr über die Weine der entsprechenden Region, Weinproduktion und die Winzer zu erfahren.

2.) Der Weintourist: Er ist weinaffiner Tourist, der in einer bestimmten Region seinen Urlaub verbringt. Vom Thema Wein möchte er am Rande berührt werden.

Nur den Weinreisenden in den Fokus zu rücken, würde aufgrund des geringen Prozentsatzes der hier untersuchten weinaffinen Touristengruppe zu kurz greifen. Diese Gruppe der Weintouristen werden als potenzielle Nutzer von weintouristischen Angeboten betrachtet, bei denen es gilt, die Erwartungshaltung zu erfüllen, um dadurch die Angebotsattraktivität einer Destination zu steigern. Daher wird der oben genannte Weintourist prioritär, unabhängig von sozidemographischen Gesichtspunkten, als Zielgruppe einer Weindestination gesehen.

\subsubsection{Die Aufgabenverteilung innerhalb einer Destination.}

Bei der Frage der Rollenverteilung zwischen Kommune, Tourismusorganisation und Weinwirtschaft in Bezug auf die Umsetzung eines Weinbaukonzeptes innerhalb einer touristischen Destination, gingen die Meinungen bei den befragten Experten auseinander: Ein Großteil der Befragten gab an, dass die Kommune treibende Kraft sein muss und diese auch die Vermarktung bündeln sollte, da hier Strukturen zusammenlaufen und so die Angebote optimal koordiniert werden könnten. Ein anderer Teil der Experten bewertet die Einführung eines Weintourismuskonzeptes ohne privatwirtschaftliches Interesse erfahrungsgemäß als nicht Erfolg versprechend und sieht eine Initiierung nur von Seiten der freien Wirtschaft als sinnvoll an. Einigkeit herrschte jedoch auf beiden Seiten darüber, dass ein Konzept für eine touristische Destination ohne Kooperation mit anderen Kommunen und Betrieben der Weinwirtschaft nicht zielführend ist.

\subsubsection{Die Erwartungshaltung von Weintouristen}

Die Auswertung der Experteninterviews zu dieser Frage orientierte sich am Ablaufmodell der zusammenfassenden Inhaltsanalyse nach Mayring [33] und den Grundlagen der Erlebnisökonomie [2]. Dafür wurden die Antworten, die die Erwartungshaltung von Weintouristen detaillierter beschreiben, in die vier Erlebnissphären von Pine und Gilmore kategorisiert. Für jede dieser Kategorien wurden Antworten aus den Experteninterviews gesammelt. Abbildung 2 zeigt die Generalisierung und Komprimierung dieser Aussagen.

Auf die Frage der Erwartungshaltung gegenüber Weingütern wurde von fast allen Experten eingebracht, dass Touristen einen zunehmenden Dienstleitungsgedanken durch zusätzliche Angebote in Form von Seminaren, Kellerführungen, Übernachtungsmöglichkeiten und Weinveranstaltungen oder auch durch ein angepasstes
Tabelle 1. Komprimierte Expertenaussagen zum Thema Erwartungshaltung von Weintouristen.

\begin{tabular}{|c|c|}
\hline $\begin{array}{l}\text { Unterhaltung } \\
\text { passive Teilnahme, Aufnahme }\end{array}$ & $\begin{array}{l}\text { Bildung } \\
\text { aktive Teilnahme, Aufnahme }\end{array}$ \\
\hline $\begin{array}{l}\text { - Außergewöhnliche } \\
\text { Veranstaltungen zum } \\
\text { Thema Wein } \\
\text { - Abendprogramme zum } \\
\text { Thema Wein } \\
\text { - Kulturveranstaltungen } \\
\text { - Geführte Weinverkostungen } \\
\text { in Weinberg und Weingut }\end{array}$ & $\begin{array}{l}\text { - Geführte Weiterbildung } \\
\text { zum Thema Wein } \\
\text { (Seminare, Führungen, } \\
\text { Kombinationen } \\
\text { mit anderen Themen) } \\
\text { - Möglichkeit, den } \\
\text { Produzenten, Produkt und } \\
\text { Produktionsmethoden } \\
\text { kennenzulernen. } \\
\text { - Zentrale } \\
\text { Informationsstellen } \\
\text { zum Angebot der } \\
\text { Destination in Form von } \\
\text { räumlichen Anlaufstatio- } \\
\text { nen und neuen Medien } \\
\text { - Einbindung neuer Medien }\end{array}$ \\
\hline $\begin{array}{l}\text { Ästhetik } \\
\text { passive Teilnahme, Eintauchen }\end{array}$ & $\begin{array}{l}\text { Realitätsflucht } \\
\text { aktive Teilnahme, Eintauchen }\end{array}$ \\
\hline $\begin{array}{l}\text { - Ansprechende } \\
\text { Landschaften, die durch } \\
\text { Weinbau geprägt sind } \\
\text { - Aufstellen von } \\
\text { Weinbaugeräten, } \\
\text { Beschilderung und } \\
\text { Kennzeichnung von } \\
\text { Gegebenheiten } \\
\text { - Weingastronomie } \\
\text { - Mit Wein assoziierte } \\
\text { Übernachtungsmöglichkeiten }\end{array}$ & $\begin{array}{l}\text { - Infrastruktur von } \\
\text { Weinwanderwegen/Rad } \\
\text { wegen in Verbindung mit } \\
\text { anderen Angeboten wie } \\
\text { Probiermöglichkeit, Kunst } \\
\text { oder Sehenswürdigkeiten } \\
\text { - Teilnahme an } \\
\text { Produktionsschritten der } \\
\text { Weinbereitung } \\
\text { - Möglichkeiten der } \\
\text { Entschleunigung, } \\
\text { Wellness } \\
\text { in Kombination mit Wein }\end{array}$ \\
\hline
\end{tabular}

Öffnungsangebot des Weingutes von den Winzern erwarten. Trends im Weintourismus, die Erwartung der Touristen zu erfüllen, sehen die Experten vor allem in zwei Aspekten: Zum Einen die zunehmende Ästhetik in Form von Wein-Architektur und zum Anderen die verstärkte Informationspolitik. Dazu gehören von einem umfassenden Internetauftritt, Beschilderung im Ort und Weinberg, ausreichend Informationsmaterial über die Region und Gemeinde, Informationsanlaufpunkte bis hin $\mathrm{zu}$ regionalen Vinotheken, in denen Winzer im Zusammenschluss Weine anbieten.

Bei der Frage nach der veränderten Erwartungshaltung von Weintouristen wurden primär folgende Aspekte angesprochen:

- gestiegene Anspruchshaltung in Bezug auf Übernachtung, Service und authentische Gastronomie

- kommunikative Wissensvermittlung und Weiterbildung im Bereich Wein

- "Interessant" gestaltete Angebote, die einen Mehrwert bieten. 


\subsection{Schlussfolgerung}

Bei der Frage "Wie wichtig ist die Thematik Wein für einen Touristen, ein spezifisches Ziel zu besuchen und wer ist der Tourist?" zeigt sich infolge der qualitativen Erhebung, dass der Weinreisende, der explizit in eine Region reist, um Wein in allen Facetten zu erleben, in der Minderzahl ist. Ein Großteil der Touristen, für die Wein im Rahmen eines Kurzurlaubes interessant ist, möchte von diesem Thema nur am Rande berührt werden. Primär stehen bei Weintouristen Entspannung und Genuss im Vordergrund, wobei diese offen sind, um "Neues Kennenzulernen" und ihr Wissen über Wein durch kommunikative Vermittlung zu erweitern.

Bei der Frage "Welche Erwartungshaltung hat ein Weintourist gegenüber dem Angebot einer Weinbaugemeinde?" zeigt sich, dass die Erwartung der Weintouristen eher klassisch und traditionell geprägt ist: Es ist festzuhalten, dass Weintouristen - wenn sie in einen Weinort kommen - ihre Präferenz auf Weinfeste und Weinveranstaltungen legen. Diese sollten allerdings mit einem Erlebniswert verbunden werden: Was passiert mit dem Wein und um den Wein herum? Der Weintourist erwartet ein Angebot an erklärten Weinverkostungen vor Ort, um damit auch sein Wissen über Wein zu erweitern - sei dies in einer Gemeinschaftsvinothek von mehreren Winzern oder beim Winzer selbst. Der Tourist möchte Schritte der Produktion miterleben. In Bezug auf die Gastronomie wird erwartet, dass diese einen direkten Bezug zu Wein aufweist. Sportlich erwartet der Weintourist Möglichkeiten zum Wandern - hauptsächlich in Kombination mit einer ausreichenden Beschilderung und der persönlichen Wissensvermittlung durch einen geschulten Wanderführer. In der Darstellung sollte die Landschaft des Weinortes durch Weinbau geprägt sein und eine ausreichende Beschilderung $\mathrm{zu}$ weinrelevanten Orten aufweisen, da der Tourist eine unkomplizierte Informationsfindung erwartet - im Idealfall auch in Form einer zentralen Anlaufstelle. Insgesamt zeigt die Befragung, dass die Erlebnissphären der passiven Teilnahme in Kombination mit Eintauchen oder Aufnahme (Ästhetik und Unterhaltung) für den Weintouristen die Prioritäten darstellen.

Bei der Frage der "Aufgabenverteilung innerhalb einer Destination, um diesen Erwartungen gerecht $\mathrm{zu}$ werden?" gaben die Experten bei der Befragung sehr unterschiedliche Antworten und Ansätze, wer die treibende Kraft in einer Destination sein sollte. Herauszustellen ist, dass die Vermarktung einer Destination nur durch eine Kooperation von Kommune und Weinwirtschaft die besseren Erfolgsaussichten hat. Die Aufteilung der strategischen und operativen Durchführung ist jedoch prinzipiell von der finanziellen, wirtschaftlichen und personellen Situation und der Motivation der jeweiligen Partner abhängig.

\section{Literaturverzeichnis}

[1] o.V., ProWein-Studie, Weintourismus liegt im Trend, (2013) http://www.prowein.de/cipp/ md_prowein/custom/pub/content, oid, 16449/ lang,1/ticket,g_u_e_s_t/local_lang
[2] J. Pine, J. Gilmore, The Experience Economy Work is Theater and Every Business a Stage, Harvard Business School Press Boston, Massachusetts (1999)

[3] J. Müller, A. Dreyer, Weintourismus: Märkte, Marketing, Destinationsmanagement - mit zahlreichen internationalen Analysen, S.9, ITD-Verlag, Hamburg (2010)

[4] H.-D. Quack, P. Hermann, Bestandsaufnahme und Bewertung des weintouristischen Angebotes in den europäischen Weinbauregionen, S.10, Europäisches Tourismus Institut GmbH, Trier (2012)

[5] C. M. Hall, G. Johnson, B. Cambourne, N. Macionis, R. Mitchell, L. Sharples, Wine tourism: an introduction, in: C. M. Hall, L. Sharples, B. Cambourne, N. Macionis, R. Mitchell, G. Macionis, Wine Tourism Around the WorldDevelopment, management and markets, Butterworth-Heinemann, Oxford, S.3 (2000)

[6] B. Cambourne, N. Macionis, C. M. Hall, L. Sharples, The future of winetourism, in: C. M. Hall, L. Sharples, B. Cambourne, N. Macionis, R. Mitchell, G. Macionis, (Hrsg.): Wine Tourism Around the World - Development, management and markets, Butterworth-Heinemann, Oxford, S.303 (2000)

[7] C. M. Hall, G. Johnson, B. Cambourne, N. Macionis, R. Mitchell, L. Sharples, Wine tourism: an introduction, in: C. M. Hall, L. Sharples, B. Cambourne, N. Macionis, R. Mitchell, G. Macionis, Wine Tourism Around the World-Development, management and markets, Butterworth-Heinemann, Oxford, S.1, 13-25 (2000)

[8] A. Dreyer, C. Antz, M. Linne, Wein und Tourismus - Trends, Synergien und kooperative Vermarktung, in: A. Dreyer, (Hrsg.): Wein und Tourismus Erfolg durch Synergien und Kooperationen, Erich Schmidt Verlag GmbH \& Co. KG, Berlin, S. 13-34 (2011)

[9] B. A. Carmichael, Wine Tourism and Sustainable Development Paper present at Leading Edge Converence, Burlington/Ontario (2001)

[10] V. Strecker, Weintourismus: Grundlagen, Konzepte \& Potenziale im Untersuchungsgebiet Südlicher Oberrhein, AV Akademikerverlag, Saarbrücken (2011)

[11] T. Bieger, C. Laesser, Tourismustrends - eine aktuelle Bestandsaufnahme, in: Jahrbuch der Schweizerischen Tourismuswirtschaft 2002/2003. St. Gallen: IDT-HSG, S. 13-38 (2003)

[12] T. Bieger, Tourismuslehre - Ein Grundriss, Haupt Verlag, Bern (2004)

[13] T. Althaus, Weintourismus. Potentiale, Markterfordernisse, Trends. Untersucht am Beispiel der Weinstadt Iphofen im "Fränkischen Weinland", Diplomarbeit, Universität Paderborn (2006)

[14] C. M. Hall, R. Mitchell, Consuming tourists: Food tourism consumer behavior, in: C. M. Hall, L. Sharples, R. Mitchell, N. Macionis, B. Cambourne, (Hrsg.): Food tourism around the world: Development, management and markets, Butterworth-Heinemann, Oxford, S. 60-81 (2003) 
[15] R. Mitchell, C. M. Hall, A. McIntosh, Wine tourism and consumer behavior, in: C. M. Hall, L. Sharples, B. Cambourne, N. Macionis, R. Mitchell, G. Macionis, (Hrsg.): Wine Tourism Around the World - Development, management and markets, Butterworth-Heinemann, Oxford, S. 115-135 (2000)

[16] S. Charters, J. Ali-Knight, Wine Tourism - A Thirst for Knowledge? in: International Journal of Wine Marketing, 12, Nr. 3, S. 70-80 (2000)

[17] R. Scheffel, Kennziffern im Tourismus am Beispiel der Deutschen Weinstrasse, Geographisches Institut der Universität Mannheim (1993)

[18] B. Cambourne, N. Macionis, Meeting the wine maker: wine tourism product development in an emerging wine region, in: C. M. Hall, L. Sharples, B. Cambourne, N. Macionis, R. Mitchell, G. Macionis, (Hrsg.): Wine Tourism Around the World - Development, management and markets, Butterworth-Heinemann, Oxford, S. 81-101 (2000)

[19] o.V. Rheinhessen-Touristik GmbH, Leitfaden: Schaufenster Weinerlebnis Rheinhessen, URL: http://www.rheinhessen.de/uploads/media/ Leitfaden_S chaufenster_Rheinhessen.pdf (eingesehen am 30.10.20 13)

[20] K. Saaler, Attraktive Weinerlebnisse durch Kooperationen, In: Das deutsche Weinmagazin, März 2013, Ausgabe 5, S.29-31 (2013)

[21] o.V. Deutscher Tourismusverband e. V., Zahlen, Daten, Fakten, http://www.deutschertouris musverband.de/fileadmin/Mediendatenbank/ PDFs/Zahlen_Daten_Fakten_2012_aktuell. pdf (eingesehen am 30.10.2013)

[22] o.V. LWG. Bayern (Bayrische Landesanstalt für Weinbau und Gartenbau), Ohne Markta nalyse geht nichts! http: //www. lwg. bayern.de/ weinbau/weintourismus/40852/linkurl_4. pdf (eingesehen am 30.10.2013) (2011)
[23] o.V. F.U.R (Forschungsgemeinschaft Urlaub und Reisen e.V.), Urlaubsarten, Forschungsgemeinschaft Urlaub und Reisen e.V., http://www.fur.de/ fileadmin/user_upload/RA_2012/ITB2012/ FUR_RA2012_Erste_Ergebnisse_web.pdf (eingesehen am 10.09.2013) (2012)

[24] G. Bleile, Zukunftstrends der touristischen Nachfrage in der Bundesrepublik Deutschland, tourism review, 43, S. 19-33 (1988)

[25] o.V. Deutscher Tourismusverband e.V. Zahlen, Daten, Fakten, http://www.deutschertouris musverband.de/fileadmin/Mediendatenbank/ PDFs/Zahlen-DatenFakten\%202011.pdf (eingesehen am 10.03.2013) (2011)

[26] K.-H. Hillmann, Handbuch der Soziologie. 4. überarbeitete Auflage, Alfred Kröner Verlag, Stuttgart (1994)

[27] H. Luft, Grundlegende Tourismuslehre, GmeinerVerlag GmbH, Meßkirch (2005)

[28] J. Swarbrooke, Sustainable Tourism Management, Cabi Publishing, Oxon (1999)

[29] E. Schmidt, Qualitätsmanagement Weintourismus: Zauberwort oder fauler Zauber?, LVWO Weinsberg http://www.1vwo-bw.de/pb/,Lde/671706 (eingesehen am 10.03.2013)

[30] E. Schmidt, Urlaubsziel Wein. So macht sich Württemberg fit für Weintouristen, LVWO Weinsberg, http: //www . lvwo-bw.de/pb/, Lde/671690 (eingesehen am 04.11.2013)

[31] K. Grötsch, Psychologische Aspekte von Erlebniswelten, in: H. H. Hinterhuber, H. Pechlaner, K. Matzler, (Hrsg.): Industrie Erlebnis Welten, Erich Schmidt Verlag, S. 69-82 (2001)

[32] G. H. Mead, Geist, Identität und Gesellschaft, Suhrkamp, Frankfurt a. M. (1973)

[33] P. Mayring, Qualitative Inhaltsanalyse. Grundlagen und Techniken, Beltz Verlag, Weinheim (2003) 